\title{
Estudio sobre Estrategia Diseño Centrado en el Humano Orientado a Impulsar la Innovación de Diseño en Guatemala -Enfocado en Salud Sector-
}

\author{
Marilú Lam Herrera \\ Estudiante de Doctorado en Ciencias del Aprendizaje, Eyes High Doctoral \\ Recruitment Scholarship 2016-2020, Universidad de Calgary, Canadá \\ Maestría en Diseño Industrial \\ Hanyang University, Corea del Sur \\ marilulam@gmail.com
}

Fecha de recepción: 15/11/2015 Fecha de aceptación: 14/04/2016

\begin{abstract}
Resumen de trabajo con conocimientos esenciales para embarcarse en proyectos innovadores dirigidos al Sector Salud.

\author{
Palabras clave \\ Diseño Centrado en el Humano, Pensamiento de Diseño, Innovación, Guatemala, Sector Salud.
}

En países industrializados las instalaciones ambientales, productos y servicios del Sector Salud mejoran constantemente, como resultado surgen formas de trabajo más eficientes. El constante desarrollo está fuertemente ligado a estudios ergonómicos de puestos de trabajo y de satisfacción del usuario, diseño y capacitación constante en el campo psicológico, físico y ambiental. Nuevas tendencias influyen en el progreso constante a través del diseño. Conceptos como: Diseño Basado en Evidencia se usan para diseñar ambientes de salud en países desarrollados. Estas mejoras evolucionan la industria de la salud. Su aplicación resulta en: mejoría del servicio, satisfacción y estabilidad laboral, mayor aprovechamiento de recursos energéticos y mejoramiento en los costos. Sin embargo, en Guatemala la aplicación de Ergonomía, Diseño Centrado en el Humano, Diseño Basado en la Evidencia u otros estudios son escasos o nulos, y cuando algunos de ellos se ha llevado a cabo, han sido realizados en zonas urbanas. El objetivo de esta investigación es resaltar la importancia de impulsar una estrategia que promueva la innovación a través de la práctica de Diseño Centrado en el Humano para que nuevas generaciones de profesionales entren en el ambiente

\begin{abstract}
:
In industrialized countries, environmental facilities, products and services in the healthcare field have been constantly improving, and as a result more efficient job performances have emerged. This constant development is strongly linked to the ergonomic study of each job, and user-satisfaction studies. Redesign and constant training, covering the psychological, physical, and environmental field. New trends influence the healthcare field, making it a non-stop effort towards progress through ongoing design development. Concepts like the Evidence-Based Design are used in the design of healthcare facilities in developed countries. These upgrades have resulted in the Healthcare Industry gaining in improved service, stability \& job satisfaction, increased energy efficiency and economic results. However, in Guatemala the application of Ergonomics, Human-Centered Design, Evidence-Based Design or other developing studies are scarce, and when some of these have been carried out, they have mostly been done in urban areas. The objective of this research is to highlight the importance of promoting a strategy to support and enhance innovation through the practice of Human-Centered Design. The training of new generations of professionals is essential to develop the Healthcare sector of the country.
\end{abstract}

\section{Key words}

Human-Centered Design, Design-Thinking, Innovation, Guatemala, Healthcare-Sector. 


\section{Bases del Estudio}

Este estudio primario tiene como objetivo demostrar la necesidad de diseñar una estrategia para impulsar la innovación en el Sector Salud de Guatemala. Aquí se muestra la viabilidad y los beneficios de implementar y dar formación práctica sobre Diseño Centrado en el Humano ( $\mathrm{DCH}$ o Pensamiento de Diseño) en diferentes niveles educativos y profesionales de Guatemala, para cambiar la estructura tradicional de pensamiento. La implementación de $\mathrm{DCH}$ puede contribuir a crear un proceso cíclico de investigación, diseño e información, que mejoraría los servicios del Sector de la Salud.

Los ambientes de Salud modernos son constantemente renovados mediante la investigación y ergonomía aplicada (Human Factors and Ergonomics Society [HFES], 2014). Las mejoras son respaldadas por organizaciones a cargo de la mejora de las instalaciones, organización, experiencias de los pacientes, y demás (ej. Planetree, Center for Healthcare Design, American Academy of Healthcare Interior Designers, Phares, 2011). Estas organizaciones utilizan el Diseño Basado en Evidencia [EBD por sus siglas en inglés] para enfrentar el reto de la innovación constante (Brown \& Schmith, Eds., 2009).

El uso de EBD proviene del enfoque de la Medicina Basada en la Evidencia [EBM por sus siglas en inglés], este es utilizado en la in- vestigación médica (McCullough, Ed., 2009). Como resultado de la inversión en renovación e innovación del ambiente de Salud, han habido grandes mejoras (Chow \& Morgan, 2007).

No sólo en las instalaciones, sino también en la satisfacción de paciente y del personal, costos, ahorro de energía, y otros (Bendewald, Berger, Birr et al., 2013). Sin embargo, en el Sector Salud de Guatemala no hay ninguna organización para la innovación, ni existen planes en el futuro cercano para desarrollarse. Por ello, es esencial para el desarrollo del Sector Salud del país que las nuevas generaciones tengan acceso a un entrenamiento apropiado.

\subsection{Entornos Salud}

El ambiente de la Salud tiene todas las características de un hotel, más los servicios de Salud. Se le considera como un 'ambiente único' (Springer, 2007, p.2), que presenta un gran reto: horas de trabajo continuas, cobertura de servicio a toda la población, regulaciones y leyes en cuanto a su funcionamiento y servicio, riesgos ergonómicos (debido a una gran variedad de actividades involucradas) uso de tecnología, personal altamente calificado, gran número de mujeres en la fuerza laboral, servicios de alimentación, servicios de limpieza regulares y de alto riesgo (UK Government, 2013), y otros, ver Fig.1. 
Figura 1

Complejidad del Ambiente del Sector Salud

\section{Características Únicas del Ambiente del Sector Salud}

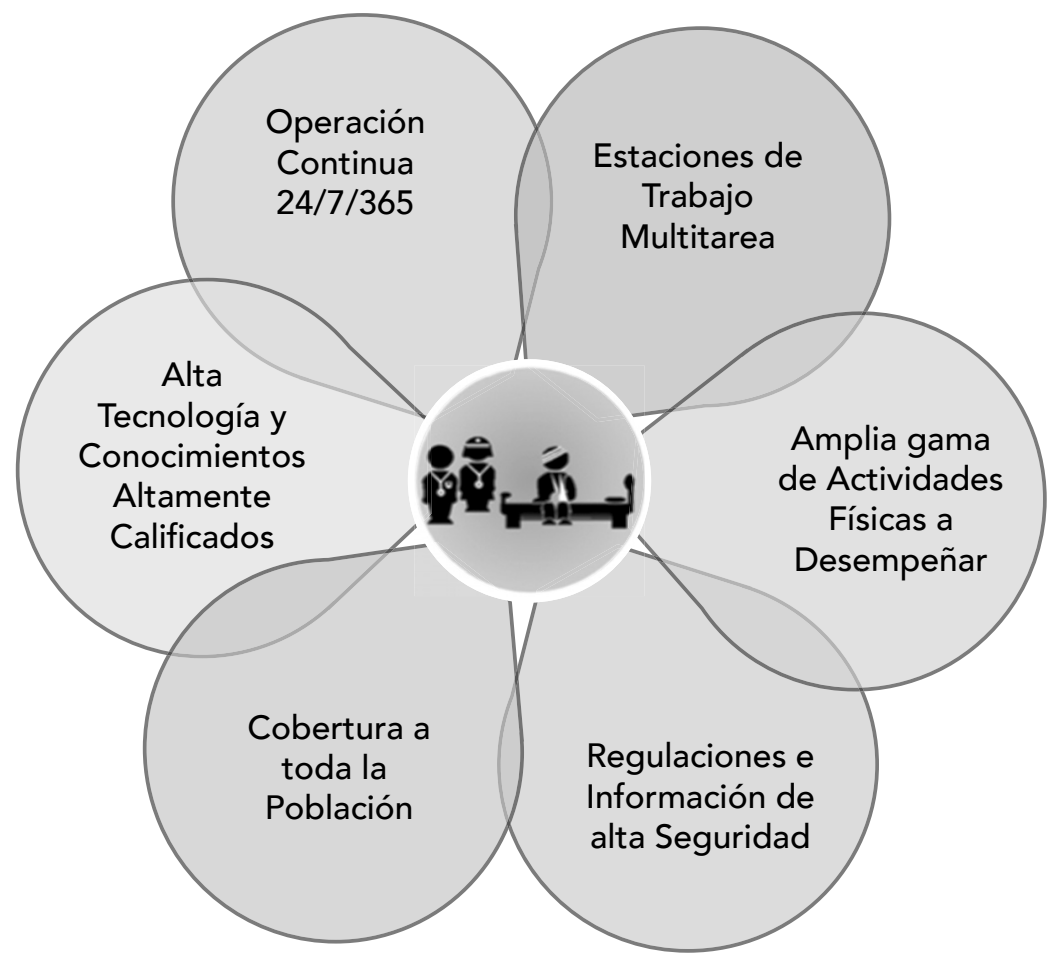

Fuente: Elaboración propia.

El ambiente de la Salud presenta puestos de trabajo con diferentes disposiciones: solo, grupal, compartido, móvil, estacionario, oficina, y otros, necesarios para la personalización del espacio. Un diseño apropiado en los ambientes de Salud mejora la calidad de los servicios, incrementa la velocidad de recuperación del paciente, brinda satisfacción laboral, mejora el desempeño y estabilidad en las estaciones de trabajo, y beneficia a las familias del los pacientes.

El diseño en el sector Salud brinda 'Diferenciación en el mercado' (Springer, 2007, p. 2), al proporcionar mejores entornos y formas de trabajo. Atrae a la gente debido a los resultados de alto rendimiento personal, crea una experiencia empática con el usuario, da mayor eficiencia en el sistema de trabajo, y más.

\subsection{Sector Salud en Guatemala}

El Sistema de Salud en Guatemala se caracteriza por estar fragmentado. Está compuesto por los sectores público y privado (Becerril \& López, 2011, ver Fig. 2). Los gastos de atención médica son alrededor de 7,8\% del PIB (Ministerio de Salud Pública, MSPAS, 2015). La situación de la salud de Guatemala tiene un gran contraste en comparación con los países desarrollados, en los que el seguro de salud cubre a la mayoría de los ciudadanos. La densidad de la infraestructura de salud y tecnologías en los hospitales en Guatemala es de 0,3 / 100.000 habitantes (OMS, 2015). Los seguros públicos y privados presentan baja cobertura (ver Fig. 3). 
Figura 2

Distribución del Sistema de Salud en Guatemala

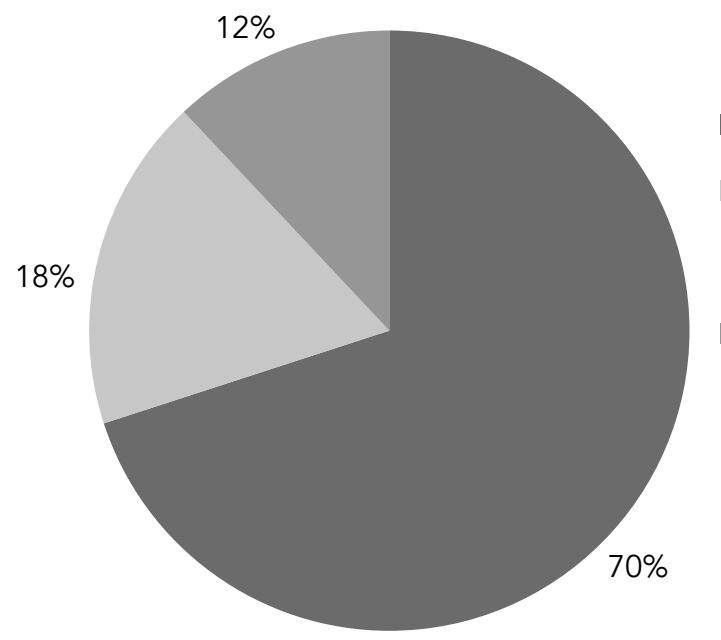

MSPAS E IGSS

Sanidad Militar del

Ministerio de Defensa

y Gobernación

Seguros Privados, ONGs,

Organizaciones Religiosas,

o sin Cobertura

Fuente: Becerril \& López, 2011.

Figura 3

Seguros en Guatemala

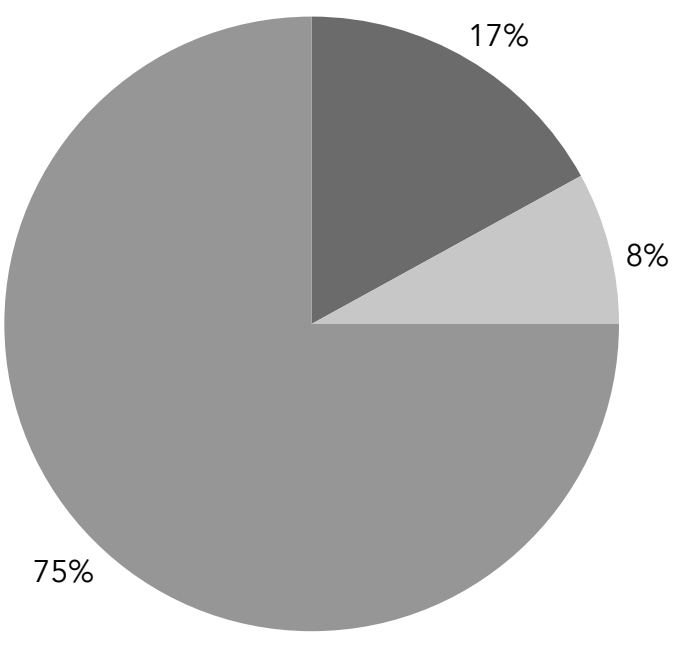

Seguro Social

Seguros Privados

Sin Conbertura

Fuente: Becerril \& López, 2011.

EI MSPAS es la entidad encargada de la designación de las políticas nacionales de salud, pero tiene baja cobertura y eficacia debido a la fragmentación del sistema y la corrupción (Transparency International, 2014). Como re- sultado, una gran parte de la población, especialmente en las áreas rurales del país, está mal atendida o desatendida. El número de personal de salud también tiene porcentajes muy bajos (ver Tabla 1). 
Tabla 1

Cobertura de Personal del Sector Salud por Habitantes en Guatemala

\begin{tabular}{|c|c|}
\hline \multicolumn{2}{|c|}{$\begin{array}{l}\text { Porcentajes de personal de la Salud / } \\
1000 \text { habitantes }\end{array}$} \\
\hline Médicos & 0.9 \\
\hline Parteras y personal de enfermería & 9.0 \\
\hline Dentistas & 1.8 \\
\hline Psiquiatría & 0.05 \\
\hline
\end{tabular}

Fuente: World Development Indicator Bank, 2012.

En un estudio realizado por Arias, Ketelhön \& Martínez (2013), se encontró que, en la región de América Central, uno de los principales problemas está a nivel administrativo. Remarcan que, a pesar de que América Latina tiene buenos resultados en la eliminación de enfermedades prevenibles, informes de la OPS muestran que la región tiene limitaciones significativas en las diferentes áreas del sistema de salud, como la sostenibilidad financiera, gerencial, organizacional, calidad del servicio, suministro de medicamentos, e incluso prestación de asistencia a toda la población. En este contexto, existe una necesidad urgente de innovación proveniente de diferentes campos.

Muchos hospitales sufren constantemente de falta de personal y suministros básicos. En los hospitales públicos, pasillos estrechos y la falta de iluminación son parte del ambiente normal. En este entorno, el personal se adapta y trabaja aun a costa de su propia salud (Andrade, 2014). Se pueden observar largas filas todos los días en las entradas de consultoría y emergencia en los centros de salud públicos, si están disponibles. Los hospitales y servicios de salud que están más equipados son menos accesibles debido al alto costo.

La gran distancia entre los pueblos y los hospitales agrava el problema. La población está dispersa, por ello los hospitales pequeños son los más comunes (ver Tabla 2). En la mayoría de estos, el personal brinda primeros auxilios. Los casos más críticos son enviados a hospitales en las ciudades más grandes, lo que afecta la vida de las familias y el personal.

Tabla 2

\section{Lista de Principales Hospitales Públicos por Capacidad de Encamamiento}

Principales Hospitales Públicos en Guatemala

Nombre

No. de

Camas

Hospital General San Juan de Dios

926

Hospital Roosevelt

791

Hospital San Juan de Dios de Occidente 364

Hospital Dres. Carlos F. Mora y F. Molina 346

Hospital Nacional Pedro de Betancourt 248

Hospital Regional de Coatepeque 202

Hospital Nacional de Jutiapa 187

Hospital Nacional de San Marcos 186

Hospital Nacional de Cobán 180

Fuente: Arias, Ketelhön \& Martínez, 2013.

En el Sector Salud de Guatemala una de las principales razones de la lentitud de los progresos, falta de servicios y cobertura de la asistencia es la corrupción que existe dentro del sistema (Courcy"s Intelligence, 2015). Según de León Duque, Defensor de los Derechos Humanos en Guatemala, menciona en el Informe Circunstanciado anual que el sistema de salud del país está dañado (De León, 2013; Andrino, De León, López y Morales, 2014). Pero, es importante mencionar que en Guatemala, existen casos de Servicios de Salud públicos en los que gracias a buenas administraciones o donaciones externas de empresas han sido muy mejorados, como ejemplo el caso de la Unidad Nacional de Oncología Pediátrica [UNOP] (aunque estos son contados). 
En lo que respecta a los pacientes privados de libertad (prisioneros), los hospitales brindan atención a los detenidos de los distintos centros de detención en el país, tanto en consulta externa como en emergencia. Ellos no tienen áreas separadas para ser hospitalizados; por lo tanto, se colocan en salas con pacientes regulares. Esta situación ha resultado en amenazas, extorsión al personal, en particular a las mujeres. La seguridad de la prisión para los detenidos es débil y no garantiza la seguridad de los pacientes ni del personal del hospital.

En Guatemala no hay ningún estudio de satisfacción del usuario de los sectores del sistema de salud, ya sea público o privado (Grover, 2011). Además, el desarrollo tecnológico, diseño o innovaciones son escasos.

\section{Diseño Centrado en el Humano}

El Diseño Centrado en el Humano (DCH), impulsa la innovación y la creación de diseños basados en el entendimiento de las personas, las tareas que realizan y sus entornos. En el, las personas están involucradas en todos los conceptos de diseño y desarrollo; idealmente debe revisarse y ajustarse con el usuario real. $\mathrm{DCH}$ involucra equipos de trabajo con una amplia gama de habilidades y percepciones porque estudia las personas y el medio ambiente en su conjunto (Goodman, Kuniavsky $\&$ Moed, 2012). Busca identificar las funciones entre los elementos del sistema en general (es decir: personas, tecnología y demás).

Cualquier proceso de diseño se realiza a través del pensamiento; se enfoca en un pensamiento continuo sobre el usuario. Por lo tanto, el diseño se puede utilizar para hacer frente a los problemas, cambiar situaciones de la vida cotidiana, dar nuevas experiencias, y mucho más. Las ideas se centran en las personas, el diseño se realiza en equipos multidisciplinarios de trabajo enfocados en crear nuevas visiones (Boy, 2013; Enterprises.org Desarrollo Internacional [IDEO], 2011). Contrario al diseño centrado en tecnología o finanzas, DCH promueve interacciones que contribuyen a hacer frente a problemas complejos a través de la integración de la tecnología, las organizaciones y las personas (Boy, 2013).

El análisis de DCH se apoya en técnicas como prototipos rápidos (utilizando materiales y objetos simples), sketches (storyboards), videos rápidos, y más. Por esta razón antes de concretar una idea, la primera etapa es para crear rápidamente modelos y bocetos. La Iteración, que es el intercambio de ideas y las pruebas con prototipos simples, es parte importante del proceso. El mostrar y recabar retroalimentación de los clientes potenciales, es muy útil para entender lo que la gente piensa sobre la manera de hacer las cosas. Sus expectativas y necesidades son una parte esencial de este enfoque (ver Fig. 4). 
Figura 4

Flujo del Diseño Centrado en el Humano

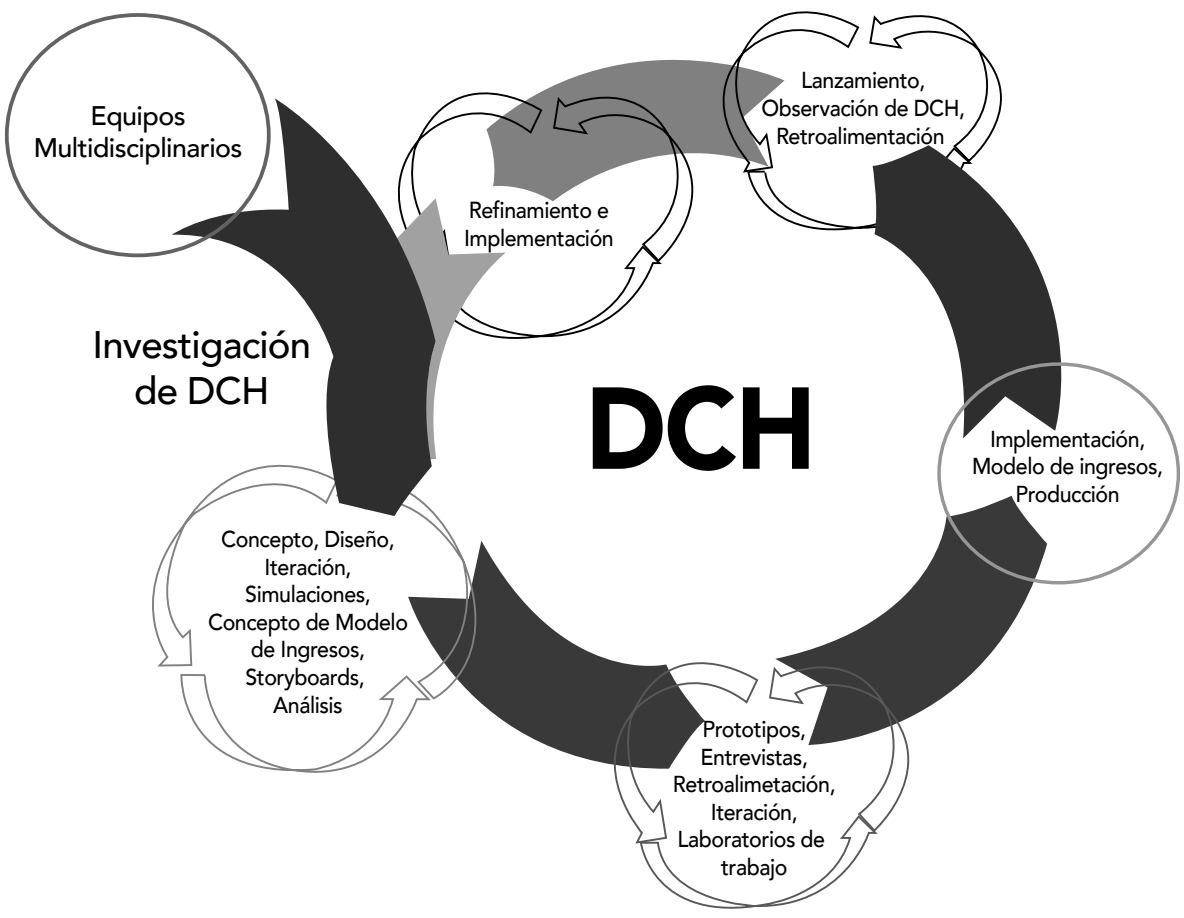

Fuente: Elaboración propia.

La investigación de DCH se centra en lo que está disponible, lo que quieren los consumidores, en entender el mercado y posibles empleados. Estar al tanto de nuevas tendencias, percepciones de las personas acerca de las situaciones, servicios y situación general. DCH toma en cuenta el mejor aprovechamiento de recursos, ayuda a observar lo que es posible hacer, y a estudiar la mejor manera de resolver los problemas de manera sostenible.

El diseño está en la vida cotidiana; las personas concretan ideas y luego se convierten en artefactos ingeniosos (en las comunidades de escasos recursos de Guatemala se ven creaciones ingeniosas, creaciones útiles construidas con materiales de segunda mano, aplicando creatividad e ingenio para ayudarse en la vida diaria).

En ambientes de recursos limitados, deben tomarse en cuenta aspectos como la 'Información de Mercado, Reglamentaciones, In- fraestructura, Conocimientos y Habilidades, Acceso a los Servicios Financieros' (Jagtap \& Larsson, 2013), ya que pueden representar barreras para el desarrollo del diseño. Con $\mathrm{DCH}$ se pueden explorar nuevas opciones sin seleccionar o separar las ideas (no se debe quedar con las primeras ideas que surjan). El objetivo es intercambiar ideas y repetir tantas ideas como sea posible (iterar) en la etapa de concepto para comprender y analizar lo que las personas piensan y necesitan.

En DCH la visión financiera de corto plazo también debe evolucionar hacia una visión centrada en el humano (spread-sheets versus reality, Boy, 2013). Lo financiero tiene que interactuar con las organizaciones en una forma innovadora (Grameen Foundation [GF], 2015). A pesar de que, DCH no es un enfoque infalible, este proporciona estrategias financieras que ayudan a los expertos a conocer las orga- 
nizaciones, productos y lo más importante, sus clientes (McKay \& Seltzer, 2014).

Independientemente del tipo de producto en el que el equipo de DCH trabaje, uno de los objetivos es diseñar el modelo de negocio y hacer que este sea rentable. Con el fin de crear valor, el equipo tiene que pensar en las capacidades disponibles, clientes, caminos financieros disponibles, y demás.

Durante la hipótesis de DCH es necesario preguntar ¿Cómo entregar? ¿Cómo agregar valor? ¿Cuáles son los puntos negativos? ¿Cuál es el riesgo del modelo? ¿Cuál podría ser la manera de enfrentar los riesgos? y más. En el análisis, el equipo tiene que ser capaz de distinguir ¿Quién es el comprador? ¿Quién es el usuario? ¿Son ellos diferentes personas? ¿Cuándo ocurre? ¿Y por qué? ¿Cuál es el contexto de la empresa o producto? Y hacer tantas preguntas como sean necesarias. DCH es muy útil para apoyar las actividades de emprendimiento (Kelley, 2007). Cuando el enfoque de $\mathrm{DCH}$ se integra en el plan de negocios, este puede proporcionar respuestas útiles.

En DCH es importante crear canales de comunicación (tecnológica, impresa, de boca en boca, y otros). Estos ayudarán a construir interacciones (brindar conexión del equipo de diseño con clientes, productores y demás). Esto con el fin de analizar lo asumido por el equipo y descubrir cuáles son las interacciones entre las partes involucradas (humanas y no humanas), es importante mantener a la vista el objetivo del diseño de manera global; y no centrarse en una cosa (ej. dinero). De lo contrario, puede causar la omisión de otros aspectos importantes que no se se ven a simple vista.

\subsection{Riesgo}

La presencia de riesgo no está sólo cuando un usuario usa máquinas o cierto tipo de automatización; está también dentro de toda una organización porque las interacciones (entre personas, tecnología, y otras) incorrectas den- tro de un sistema (ej. Accidente de fuga de Cesium-137 en Goiania, Brasil, en 1987 maquinaria de radioterapia abandonada causó un accidente desastroso en una comunidad, Lawnorder, 2003) puede desencadenar accidentes fatales.

Cuando se estudian los factores que conducen al accidente, la rutina y la complacencia son parte de las primeras causas. Es necesario entender cómo las personas se involucraron en accidentes. La práctica de la simulación durante el proceso de DCH ayuda a entender el posible tipo de riesgos, ya sean voluntarios o involuntarios (Boy, 2013, p. 50). Las variantes de riesgo pueden ser ilimitadas (ya que dependen de la reacción " $X$ " de cada persona a las diferentes situaciones, para tomar decisiones las personas utilizan no sólo sus conocimientos, sino también pensamientos, emociones y más). Las estrategias de simulación para hacer frente a los casos de riesgo no son del todo infalibles; sin embargo, su práctica brinda mejores estrategias.

\subsection{Equipos multidisciplinarios}

El carácter multidisciplinar de los equipos en DCH es indispensable debido a que el análisis requiere el estudio del problema y propuestas de soluciones provenientes de diferentes campos del conocimiento, experiencia y habilidades. El equipo tiene que ser interdisciplinario y proactivo, con personas capaces de analizar el sistema en su conjunto desde diferentes perspectivas hacia una meta común. Contrario a centrarse en usabilidad, el equipo de DCH se centra en lo que desconocen (ej. oportunidad de negocio, medio ambiente, producto, y demás). Deben recoger información de lo desconocido y analizarla de forma repetitiva. El equipo interdisciplinario es el que tiene la capacidad de agregar valor a la creación.

Por lo tanto, como es esencial tener presente que todas las ideas son valiosas, el líder de $\mathrm{DCH}$ debe tener en cuenta la personalidad 
de las personas en el equipo, y proporcionar espacio para que todos transmitan sus ideas durante las sesiones de diseño (Caín, 2013). En la práctica de DCH es mejor empezar de cero y con muchas cosas por entender porque en el curso de iteración las ideas maduran, se refinan y se adaptan hacia la meta.

El equipo trabaja con plazos cortos de tiempo, expone las ideas a las personas, habla con expertos técnicos, clientes, usa escenarios para entender, hace prototipos para volver a arreglar y re-diseñar tantas veces como sea posible. Durante el proceso de ideación, es importante no aferrarse a las primeras ideas; mantener una sola idea por mucho tiempo puede conducir a la ceguera o el engaño en el proceso. Una cantidad conveniente de tiempo para la reflexión sobre diferentes ideas puede variar de dos a tres semanas (Novo Ed, 2015), pero también dependerá de las necesidades especiales del diseño.

\subsection{La experiencia y $\mathrm{DCH}$}

El aspecto de la experiencia es fundamental porque el diseñar escenarios precisos requiere habilidades y conocimientos, sobre como gestionar operaciones complejas y emergencias. Los expertos son los únicos capaces de contribuir con el pensamiento crítico basado en la experiencia para hacer frente a las tareas difíciles y complicadas.

El surgimiento de organizaciones automatizadas, ha dado como resultado más tecnología que controla operaciones, convirtiendo la función humana en administrador en lugar de operador. En las organizaciones automatizadas la experiencia, cooperación, coordinación y comunicación son cruciales. Toda la organización debe dirigirse al mismo objetivo; de lo contrario los elementos (humanos y máquinas) estarán trabajando ciegamente (ej. el 6 de julio 2013, 'Accidente Fatal de Asiana en San Francisco', los pilotos no entendían plenamente los controles, Bachman, 2014). El punto principal del éxito de un producto o servicio es tener controles simples (complejidad externa sencilla); sin embargo, esto se obtiene a través de alta complejidad interna.

En el DCH, el equipo de trabajo es el que hace la diferencia. Los integrantes deben ser personas con experiencia, motivados, polivalentes, competentes, trabajando hacia un mismo objetivo y visión. Un equipo con estas características genera resultados positivos. Para poner un ejemplo, tenemos el uso que se le ha dado al teléfono celular como instrumento para resolver problemas en diferentes partes del mundo. Las interacciones que se logran a través de ellos están cambiando vidas. Estos aparatos pueden ser tan útiles como la experiencia, formación y creatividad involucrada. Hay ejemplos que han sido registrados en África, Enclaves de la India y Bangladesh (Ogunlesi, 2012; BBC Monitoring, 2015), Mali (Proyecto Pésinet, Jagtap \& Larsson, 2013) y Brasil (Esfuerzos por salvar la selva tropical, White, 2014), por mencionar algunos ejemplos.

Cuando la vida está en juego, además de los aspectos financieros (ej. Caso del Contrato de Servicios de Diálisis en Guatemala, Malkin, 2015), las organizaciones deben tener en cuenta aspectos importantes, como: experiencia, competencia, líderes socio-técnicos, compromiso y la motivación de los trabajadores, y claro rol de los elementos que interactúan en el sistema. Es necesario tener en cuenta que el error humano es un factor constante, y más importante, que el ser humano (basado en su experiencia) es el que enfrenta cualquier fallo inesperado.

Nota: Contrario al pensamiento tradicional existente entre los profesionales de algunos países en desarrollo que opinan que el paciente no debería tratarse como Cliente, desde el punto de vista de Design Service, Human-Centered Design y Healthcare Design, el paciente ES un cliente, al igual que sus familias y las comunidades para las que se desarrolla 
el diseño. Para innovar es de vital importancia centrarse en sus necesidades. De allí que innovar se convierte en una actividad compleja, por que se requiere un gran esfuerzo, tiempo y actitud positiva para cambiar la mentalidad de las personas y lograr dar cabida al pensamiento de diseño y desarrollo.

\section{Metodología de investigación}

En el entorno de diseño, conceptos como: Pensamiento de Diseño o Diseño Centrado-Humano son conocidos. Sin embargo, es importante conocer el grado de conocimiento que la población guatemalteca (además de los diseñadores) tiene en esta materia, con el fin de identificar la disponibilidad e interés en aprender acerca de las estrategias para innovar, y medir la complejidad percibida de los guatemaltecos con respecto al Sector Salud.

Los datos estadísticos se obtuvieron de entidades nacionales e internacionales, agencias gubernamentales, informes anuales y otros. También se hizo uso de recursos informales, como agencias internacionales de noticias, conferencias, revistas, artículos académicos y más. Además, se realizó una encuesta en línea de veinte preguntas. Esta fue revisada y evaluada a través de una prueba piloto con un profesor de la Facultad de Diseño de la Universidad de Hanyang en Corea del Sur, y otras seis con profesionales guatemaltecos.

La encuesta fue diseñada para sondear sobre la visión general acerca de los servicios de salud y el conocimiento que los guatemaltecos tienen en cuanto a DCH, con el fin de evaluar su posible aplicación en la innovación del Sector Salud del país. Adicional a ello, se realizaron seis entrevistas vía Email con expertos guatemaltecos, provenientes de diferentes especialidades. Esto a fin de conocer la complejidad percibida para la estrategia desde un análisis multidisciplinario.
Teniendo en cuenta que en Guatemala las encuestas de satisfacción del usuario del Sector Salud son escasas, se incluyeron preguntas que abordan el ambiente de la Salud. Se indagó acerca del conocimiento sobre $\mathrm{DCH}$ e ideas que podrían beneficiar al sector de la salud del país. Los resultados se incluyeron en gráficas y tablas estadísticas. Esta información es importante para demostrar la necesidad de desarrollar una estrategia para innovar el Sector Salud (objetivo principal del estudio).

- El éxito es una buena mezcla de conocimiento, entusiasmo, confianza, coraje, perseverancia y la asunción de riesgos bien pensada. Todo esto es centrado en el humano. - ["success is a good mix of knowledge, enthusiasm, confidence, courage, perseverance, and well-thought- out risk-taking. All this is human centered."] (Boy, 2013).

\section{Fuentes, Evidencia y Soporte del Estudio}

La encuesta fue dirigida a la población guatemalteca en general, aunque debido a la distancia (fue realizada desde Corea del Sur) y a que la herramienta utilizada fue en línea, quedaron sectores de la población que no tuvieron acceso a ella. Sin embargo los resultados dan una premisa de la situación general y genera un incentivo para la realización de un estudio más a fondo en el futuro. Fue respondida por ochenta y cinco personas de diferentes niveles educativos, económicos y sociales.

Se obtuvo información general sobre la calidad de los servicios de salud y las necesidades percibidas. También se obtuvo el grado de conocimiento sobre innovación, así como ideas y sugerencias que los guatemaltecos tienen sobre la mejora de los servicios de Salud. Los resultados se muestran en las sub-secciones relacionadas con el tema en esta sección (ver Tabla 3 y Figs. 5 y 6). 


\section{Tabla 3}

Demografía de la Encuesta

\section{Descripción de la Muestra}

\begin{tabular}{lccc} 
& Mujeres & Hombres & Total \\
\hline Participantes & 60 & 25 & 85 \\
\hline Edad promedio & 37 & 32 & 34 \\
\hline $\begin{array}{l}\text { Ingreso familiar } \\
\text { promedio mensual en } \\
\text { dólares }\end{array}$ & 1,170 & 1,148 & 1,159 \\
\hline
\end{tabular}

Fuente: Elaboración propia.

Figura 5

\section{Profesión u Oficio de los Encuestados ( $n=85$ )}

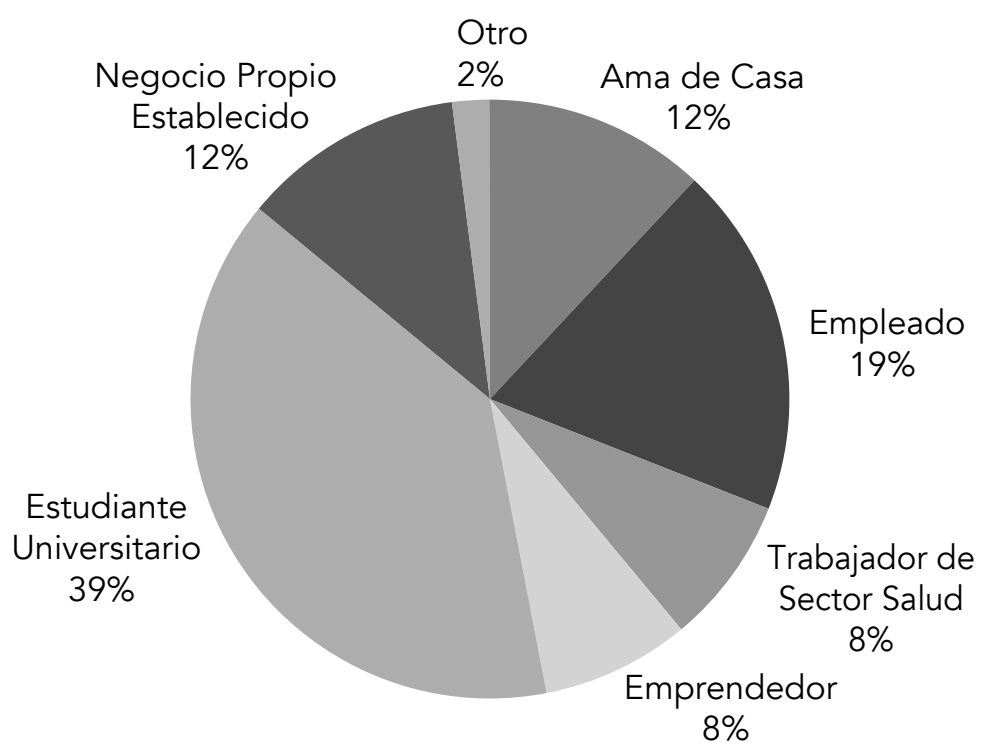

Fuente: Elaboración propia. 
Figura 6

Nivel Educativo de los Encuestados $(n=85)$

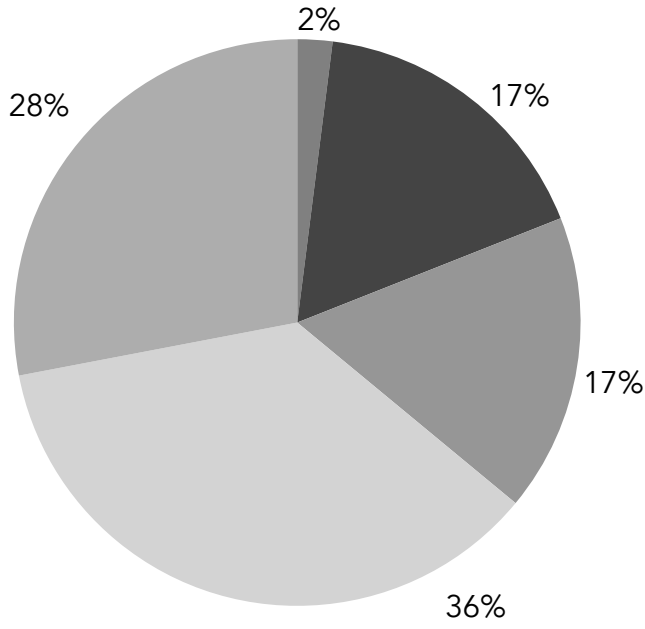

Diversificado

Técnico Especializado

Licenciatura

Maestría

Doctorado

Fuente: Elaboración propia.

\subsection{Resultados de la encuesta}

Con el fin de demostrar que existe la necesidad de implementar una estrategia para promover la innovación en Guatemala, era necesario reunir información sobre la percepción de la población guatemalteca en relación con: la situación actual de los servicios de salud, el desempeño de la salud relacionados con el personal, y el orden de prioridad percibido en lo que respecta a la mejora de los servicios de salud en el país.

Las áreas que los participantes perciben como "Muy deficiente" son: 'Suministros de medicamentos y otros insumos' (50.6\%). Le sigue: 'Tiempo de espera para las citas' y 'Organización y Administración de Recursos' con el $47 \%$ y $44.6 \%$ respectivamente.

En la clasificación de la categoría de "Deficiente": 'Seguridad' y 'Precios accesibles' con resultados iguales (53\%), siguiendo 'Instalaciones y Equipo' y 'Tiempos de espera para pasar a la consulta' ambos con $45.85 \%$. Y 'Tiempo de espera para ser atendido en emergencia' en esa misma categoría $41 \%$.

Es importante tener en cuenta que los servicios públicos y privados no se estudiaron por separado (los resultados pudieron verse afectados debido a la herramienta utilizada: servicios de encuesta vía internet de Google Forms). Sin embargo, en los resultados (tanto en los servicios públicos como privados) existe la percepción de mala calidad en los servicios. La preferencia de uso de los servicios (públicos o privados) puede verse afectada también por la percepción de la deficiencia en el sector público (algunos encuestados expresaron que a pesar de que pueden utilizar los servicios públicos, debido a su mala calidad, prefieren el privado) (ver Fig. 7) 


\section{Figura 7}

Respuesta de los encuestados acerca de Cuál es el Servicio de Salud que Utilizan

Servicios de Salud utilizados

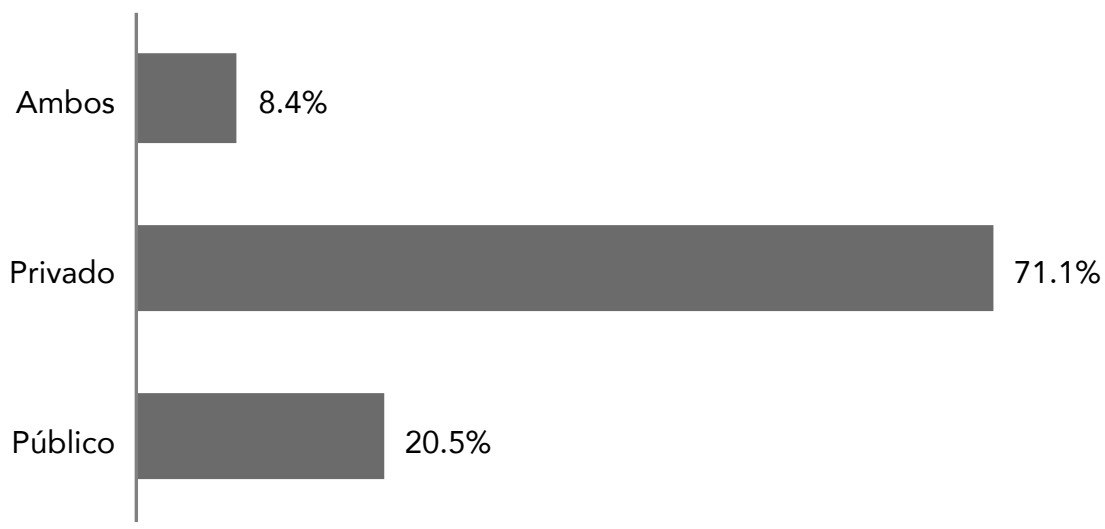

Fuente: Elaboración propia.

Nota: el aspecto de 'Seguridad' se refiere a la violencia en general que está afectando al país (amenazas, hostigamiento, extorsión y otros, que han sido reportados por los pacientes y el personal).
En la percepción general del Servicio de Salud, dos categorías son predominantes: "Deficiente" con $42.1 \%$, y "Muy deficiente" con 34.8\%. La categoría de "Excelente" fue casi nula (0.5\%). La visión general se muestra en la Fig. 8.

Figura 8

Percepción General de los Encuestados sobre los Servicios de Salud

Percepción General del Sector Salud

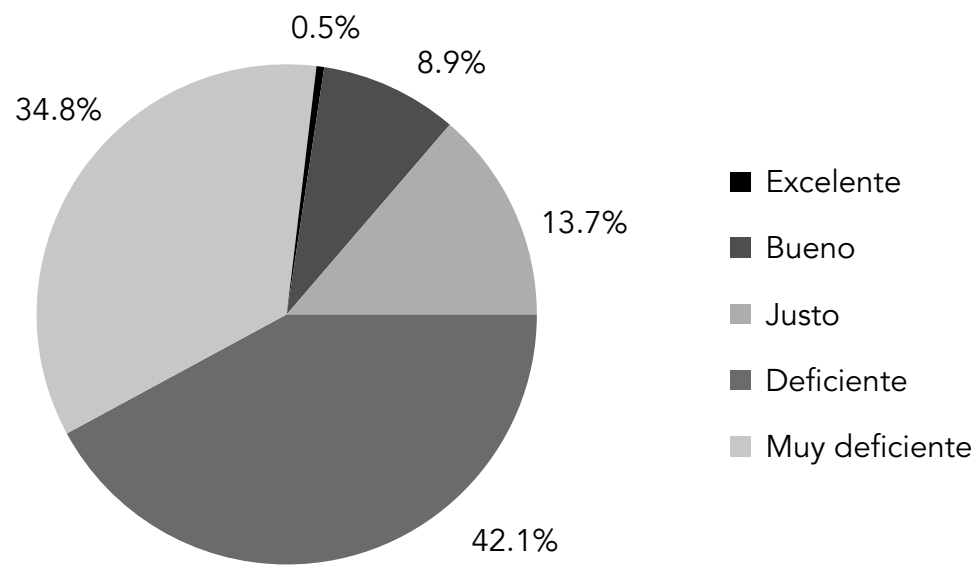

Fuente: Elaboración propia. 
En cuanto al Desempeño del Personal. En la categoría de "Muy deficiente": 'Administración y Recepción' y 'Seguridad' obtuvieron el rango más alto con $20.5 \%$, seguido por 'Limpieza' y 'Enfermería' con $16.9 \%$ y $15.7 \%$ respectivamente. En la categoría de "Deficiente": 'Recepción y Administración' con $41 \%$, seguido 'Emergencia' 32.5\%. 'Rehabilitación' y 'Seguridad' con rangos muy similares (26.5\% y $27.7 \%$ respectivamente). Es importante notar que, a pesar de las deficiencias percibidas en el entorno global la categoría de "Justo" en cuanto al desempeño del personal obtuvo 33.9\% (ver percepción general en la Fig. 9).

Figura 9

Percepción General de los Encuestados sobre el Desempeño del Personal del Sector Salud

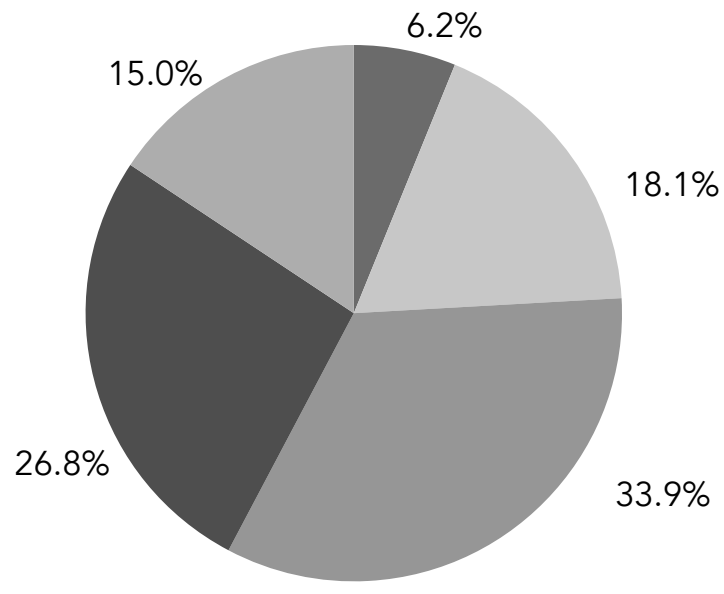

Excelente

Bueno

Justo

Deficiente

Muy deficiente

Fuente: Elaboración propia.

En términos de la prioridad para mejorar el sector de la salud en Guatemala, hay cinco aspectos que se destacan como "Muy urgente". El primero, el 'Abastecimiento de medicinas e insumos' con un $84.3 \%$ de liderazgo, luego 'Tiempo de espera para ser atendido en emergencia' con $60.2 \%$, finalmente 'Instalaciones y Equipo' y 'Acceso a los servicios de salud' ambos con $56.6 \%$ (ver percepción general en Fig. 10). 
Figura 10

Percepción General sobre la Urgencia de Mejorar el Sector de la Salud

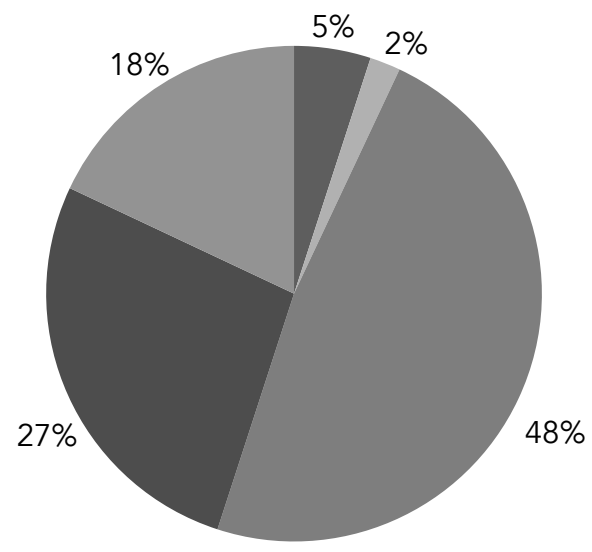

Muy urgente

Urgente

Necesario

Sería bueno

Puede esperar

Fuente: Elaboración propia.

\subsection{La Percepción General de los Guatemaltecos sobre DCH}

A través de la encuesta se encontró que los guatemaltecos tienen poco conocimiento acerca de DCH (ver Fig. 11). Ello puede deberse a que los canales de transmisión son escasos.
Esto, a pesar de que en la actualidad el conocimiento sobre DCH está accesible en Internet, incluso algunos de los cursos son gratuitos (NovoEd, 2015). Estos son cursos simples en línea con guías rápidas para dirigir el trabajo que incluyen cierto apoyo para su consulta.

Figura 11

Respuesta de los Encuestados a la Pregunta ¿Has oído alguna vez acerca de Diseño Centrado en el Humano o Pensamiento de Diseño?

Conocimiento sobre Diseño Centrado en el Usuario

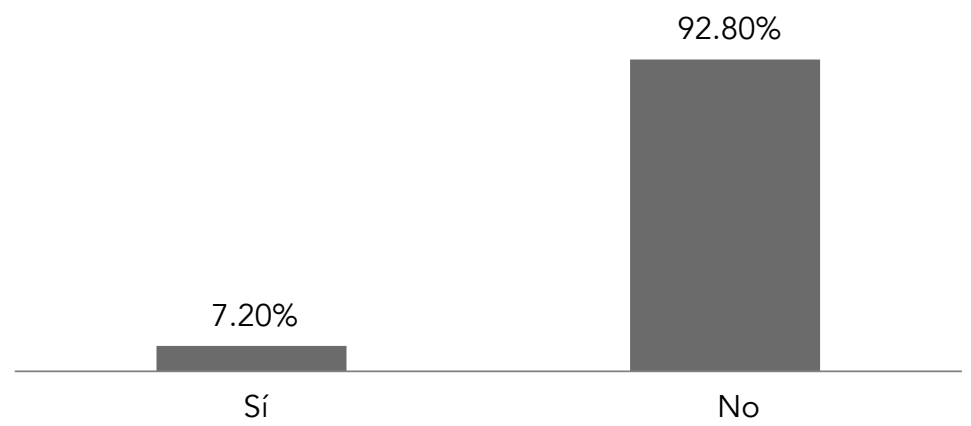

Fuente: Elaboración propia. 
Los encuestados que conocían sobre DCH reportaron (en mayor proporción) haberse enterado de persona a persona. Cabe destacar que ninguno de los encuestados informó haberse enterado a través de revistas o libros. Y la radio, que es uno de los medios de comunicación más populares en el país, no fue mencionada. Los resultados de estos datos están en la Fig. 12.

Figura 12

Respuesta de los Encuestados sobre

¿Cómo se enteraron acerca de Diseño Centrado en el Humano?

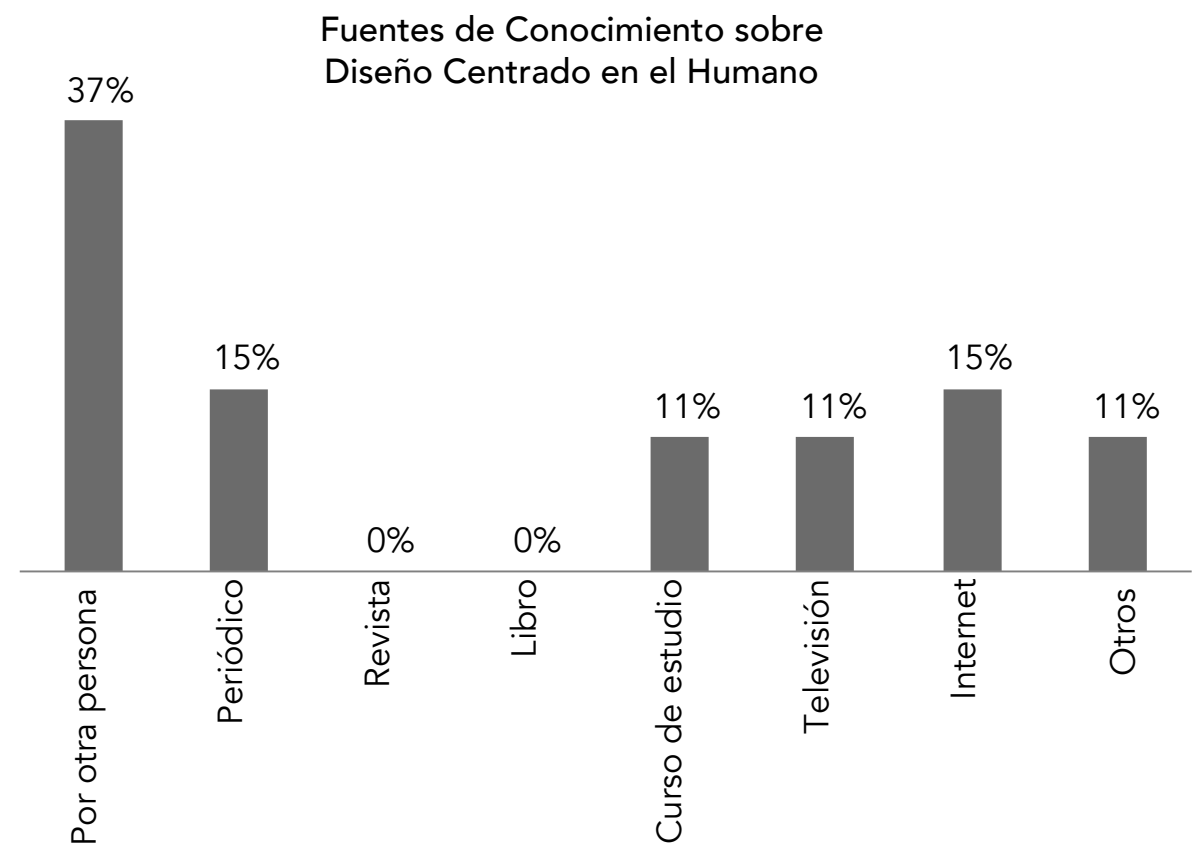

Fuente: Elaboración propia.

La disposición general de los encuestados en cuanto a aprender sobre innovación es positiva (87.2\%). Esto, en gran parte podría deberse a que muchos guatemaltecos subsisten con negocio propio y están constantemente en actividades de emprendimiento (GEM, 2014). Otra razón es la baja oferta de empleo que existe en el país. Es importante señalar que, el tiempo promedio más seleccionado se encuentra entre '1 y 2 horas' $30.1 \%$ y $37.3 \%$ respectivamente). Esto es positivo, porque este tiempo es el promedio ideal de uso para capacitación en laboratorios de $\mathrm{DCH}$ (ver Figs. 13 y 14). 
Figura 13

Interés para Aprender sobre Innovación

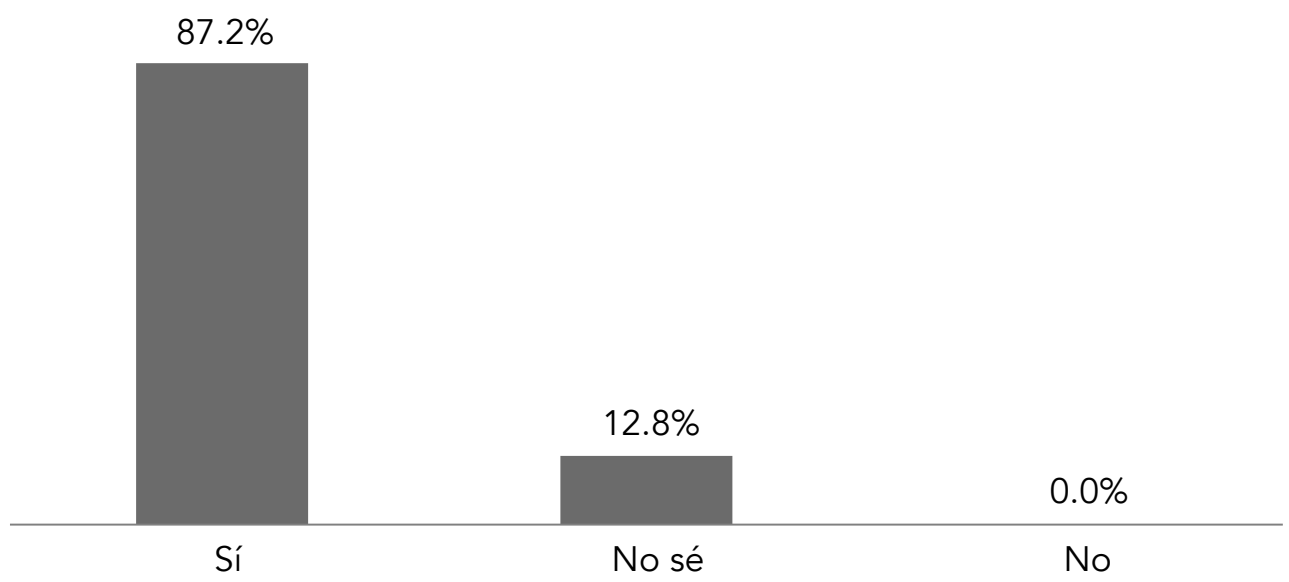

Fuente: Elaboración propia.

Figura 14

Respuesta de los encuestados acerca de la Disponibilidad de Tiempo para Aprender sobre Innovación

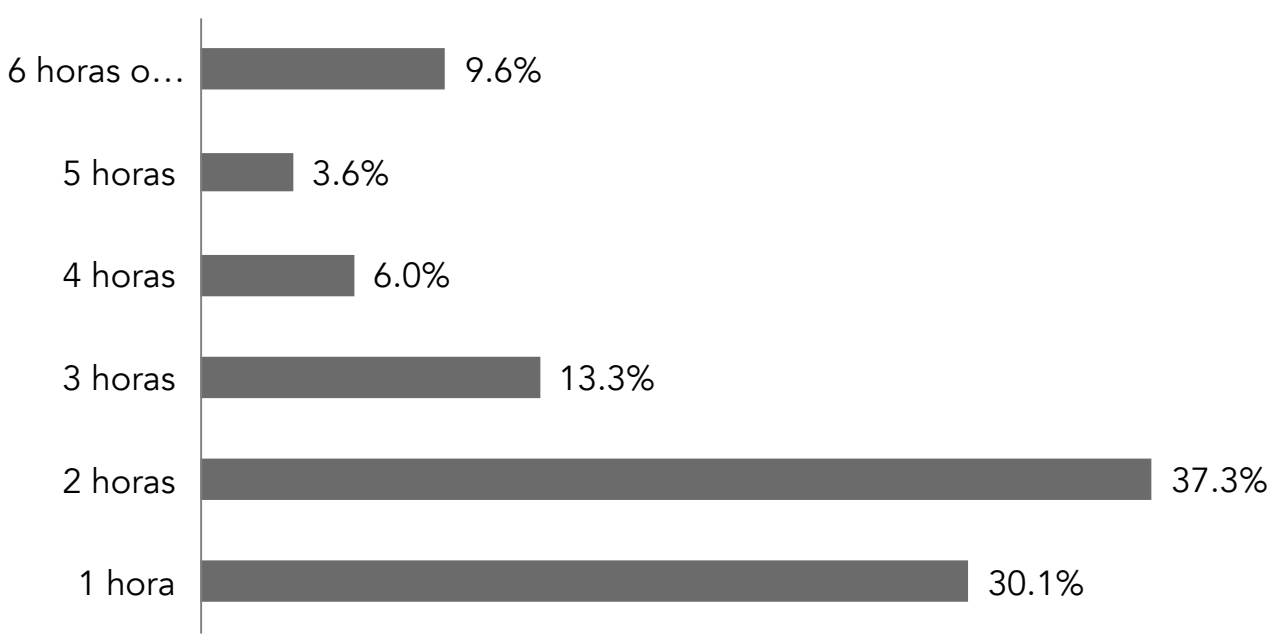

Fuente: Elaboración propia. 
En el enfoque de DCH (aunque requiere trabajo de investigación individual) el trabajo en equipo es muy importante; por lo tanto, fue importante indagar acerca de la disposición de los guatemaltecos respecto a su voluntad de trabajar en equipo. Estos resultados muestran que la gran mayoría, tienen una predisposición natural a este esfuerzo conjunto. Las categorías 'Me gusta mucho' y 'Me gusta' fueron las más altas (48.8\% y $38.4 \%$ respectivamente, ver Fig. 15 ).

Figura 15

Opinión de los Encuestados acerca del Trabajo en Equipo $(n=85)$

\section{Opinión sobre el Trabajo en Equipo}

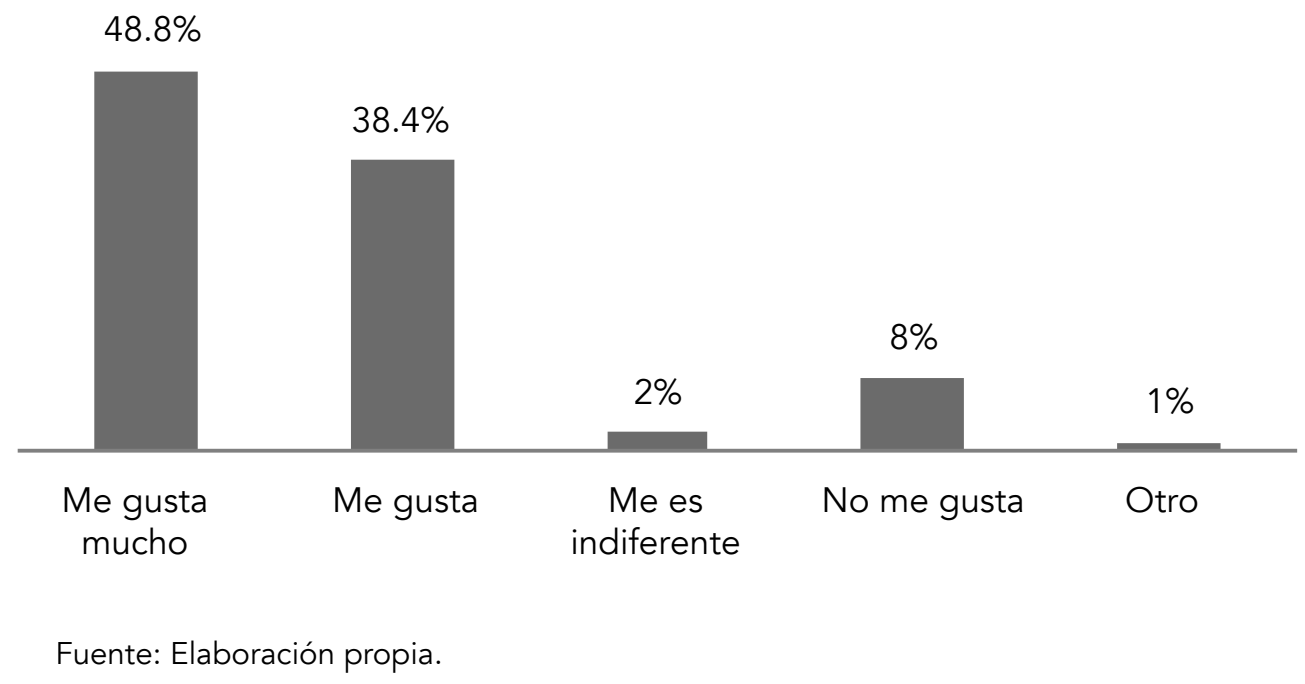

El ambiente guatemalteco está lleno de retos que enfrentar: analfabetismo, violencia, corrupción y otros. El contexto en el que se debe impulsar una estrategia para innovación en Guatemala ha sido descrito en la primera sección de este estudio. Pero, para los fines específicos de aprendizaje y formación sobre DCH, fue necesario conocer qué obstáculos se perciben como las mayores limitaciones para participar en aprender. Se encontró que, dentro de los principales obstáculos para participar en este esfuerzo están: 'Tiempo' y 'Dinero' $(60.5 \%$ y $24.4 \%$ respectivamente). Sin embargo, es importante tener en cuenta, que debido a que la encuesta se realizó a través de la Internet, la percepción de la limitación de 'Acceso a Internet', pudo verse afectada, ver Fig. 16. 
Figura 16

Opinión de los Encuestados sobre su Mayor Obstáculo para Aprender

Limitaciones para el Aprendizaje

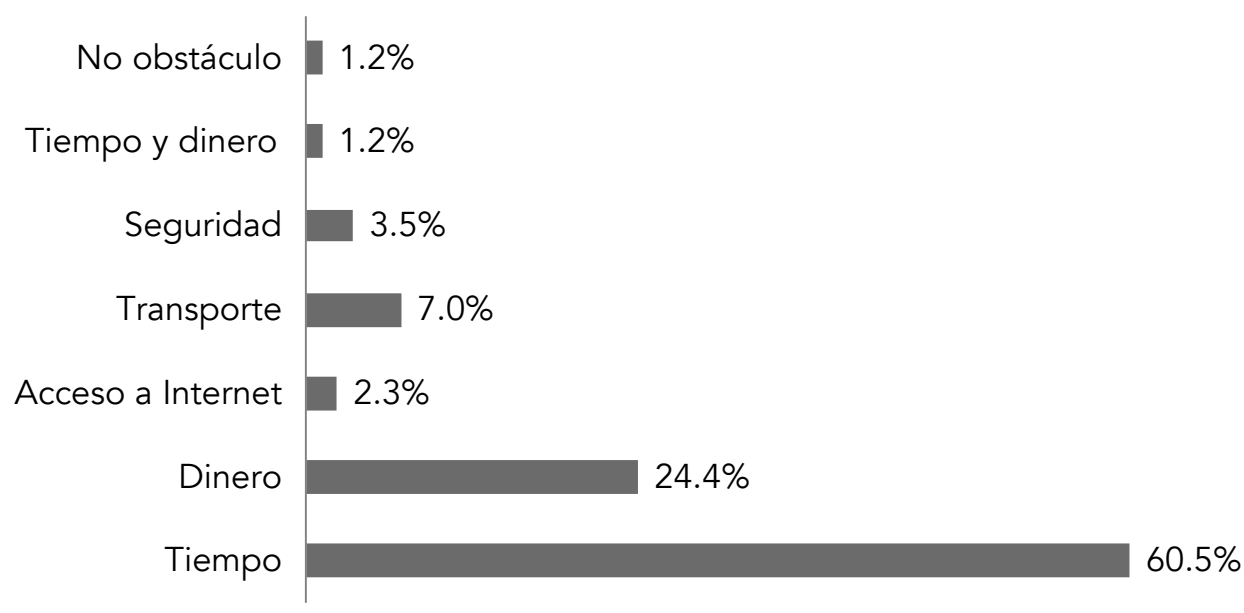

Fuente: Elaboración propia.

Link del estudio completo:

https://drive.google.com/file/d/OBwTwcltveKOmWWtOaUdmSVFEVTQ/view?usp=sharing

\subsubsection{Complejidad Percibida en Sector Salud de Guatemala}

El análisis de la complejidad de la implementación de una estrategia de innovación en el Sector Salud de Guatemala se llevó a cabo con la colaboración del Dr. Juan Ricardo Ortega Moreno, Especialista en Medicina Interna, Sanatorio CEMS, Guatemala. Desde su punto de vista (como un experto en el campo de la Salud), las principales limitaciones se pueden encontrar en diferentes niveles: gubernamental, falta de personal calificado, poco uso de la ayuda internacional, y otros.

En lo que respecta al gobierno, se observa una barrera financiera. Por ejemplo, el presupuesto asignado para el 2015 al Ministerio de Salud fue Q5,647.22 millones, que es el 7.8\% del PIB (MSPAS, 2015). Este es demasiado bajo para cubrir todo el país. Por lo tanto, el plan de ejecución se enfoca en prioridad sólo las necesidades básicas, sin dejar presupuesto para invertir en nuevas estrategias. En el sistema público, es común dar gerencias y puestos principales por parentesco o amistad; en lugar de dárselos a personas con la titulación o experiencia profesional necesaria. Y como resultado hay falta de personal calificado en este sector.

Otro punto a destacar es la falta de sub-especialidades. A este respecto, es importante mencionar los avances que se están realizando en el Africa Sub-sahariana en donde las universidades trabajan para encontrar soluciones para el país, se crearon programas de certificación única ["Dual infection with oncogenic human papilloma virus (HPV) and human immunodeficiency virus $(\mathrm{HVI})$.... Visual inspection with acetic acid (VIA) has been shown to be an effective means for detecting cervical pre-cancer ... treatment with cryotherapy for appropriate VIA positive lesion"; solutions "Driven 
by Need, not by Desire", Cu-Uvin, 2015]. Esta certificación respalda el procedimiento para combatir la "infección dual con el virus oncogénico del papiloma humano [VPH] y el virus de la inmunodeficiencia humana [VIH]

En el sector privado, existen algunas innovaciones en el campo de la salud en Guatemala (ej. Leveraged Freedom Chair, Innovation Lab, 2015). El punto es que, aunque la medicina en sí misma es un campo enfocado en la salud de las personas, es también un negocio. Este es un importante punto de motivación que se puede utilizar para mejorar la calidad de servicio del paciente/cliente, motivar y generar no sólo un mayor acceso a los recursos sanitarios (accesible para la mayoría), sino también llevar a cabo una mejor investigación acerca de cómo enfrentar los desafíos del país, crear más puestos de trabajo, mejores servicios y en general hacer progresar todo el sector.

\subsubsection{Papel de las Universidades e Institutos Técnicos en la Estrategia de DCH para la Innovación}

En Guatemala, desde el nivel de licenciatura hasta el más alto grado de la universidad se realizan trabajos de investigación. Las universidades tienen diferentes programas en los que los estudiantes participan en función de la ideología de la institución (ej. la Universidad Francisco Marroquín, Guatemala National Team, GEM, 2013; Universidad del Istmo [UNIS], FORHUM, Formación Humanística, UNIS, 2015 y otros). Además, muchas personas finalizan su educación en el nivel Diversificado; por lo tanto, obtener certificaciones adicionales u obtener Diplomas de especialidades técnicas es bastante frecuente.

En Guatemala el apoyo para los temas de la innovación no tiene suficientes recursos financieros, solo cuenta con la aceptación y voluntad de las personas para mejorar y colaborar.
Por lo tanto, a fin de evaluar la complejidad percibida en el entorno educativo de Guatemala en relación a innovar a través de $\mathrm{DCH}$, se entrevistó a la Licda. María Teresa Bustamante de Molina, Directora de Diseño Industrial con Especialización en Vestuario, UNIS.

De Molina encontró de gran interés el DCH. Ella compartió el caso de un trabajo de investigación que se hace en el departamento (para demostrar el potencial que la estrategia podría tener para el país). Esta investigación es llevada a cabo por un grupo de estudiantes de Diseño Industrial de Vestuario en conjunto con una institución de salud que supervisa los niños con problemas de piel llamada Xerodermia Pigmentosa, esta se transmite de padres a hijos (producto de uniones consanguíneas: entre hermanos, padres con hijas o parientes cercanos).

Se investiga sobre textiles con la propiedades de bloqueo de rayos ultravioleta, se desarrolla diseño de ropa y se da una solución para los niños. El resultado es la creación de Ropa UV-Block, ya que el estado de los pacientes se agrava cuando se exponen al sol. Los estudiantes han hecho una investigación para encontrar los textiles apropiados. Tanto, la institución de la salud como los estudiantes trabajan con recursos económicos limitados, sin embargo, en opinión de la Licda. Molina, la manera de trabajar con poco o nada de recursos, es a través del trabajo conjunto con instituciones que deseen colaborar.

Desde su punto de vista, es necesario desarrollar esta estrategia, sobre todo para cuestiones de salud y seguridad. Ella ve viable implementar el DCH en el ámbito universitario. Si la estrategia está bien fundada, las universidades pueden adaptarse y ofrecer especialidades, planificar los cursos necesarios en el plan de estudios para aumentar la capacitación. Es posible y necesario. 


\subsubsection{Complejidad Percibida para Introducir la Innovación en Sector Salud a través Proyectos Comunitarios y ONGs}

Es frecuente que en Guatemala se lleven a cabo proyectos de desarrollo por parte de ONGs. Por ello, la autora consideró necesario contar con la opinión de un experto en proyectos de desarrollo comunitario sobre la complejidad percibida sobre una estrategia para innovar. El experto entrevistado fue la Técnica Regional del Proyecto Damaris Raquel Estrada Toraya (el nombre de la ONG para la que trabaja se omitió debido a los reglamentos internos de la organización).

En opinión de la Licda. Estrada, hay una sensación general de cambio radical, esperanza en las nuevas autoridades de gobierno y fortalecimiento de las estructuras de los sectores gubernamentales (sensación general debido a los recientes acontecimientos político-sociales, \#Justicia Ya, 2015). Sin embargo, en su experiencia personal, el control externo de la ejecución del proyecto sigue siendo importante. Por ello las entidades internacionales establecen sus propias políticas y estrategias dentro de sus programas de desarrollo con el fin de evitar el fraude.

Actualmente, cuando una institución internacional da apoyo, tiene que empezar por la formación de sus empleados sobre el servicio y la gestión, además de instalar mecanismos de auditoría para sus procesos. Se han dado casos en que la ayuda exterior se concede directamente al gobierno del país receptor y los fondos son mal invertidos o desviados, esto genera la práctica del "Jineteo del dinero" (que es poner el dinero a trabajar en financieras para generar intereses y multiplicar ganancias). Eso frena y obstaculiza el progreso, disminuye la transparencia, eficiencia y la respuesta pro-activa de las personas involucradas.

Estrada Toraya ve un gran potencial en Guatemala para proyectos innovadores. Las perso- nas en las zonas rurales (su área de especialización) son muy cooperadoras y pro-activas con las instituciones que les dan apoyo a través de los proyectos. Están dispuestos a compartir información para adaptar el proyecto a sus realidades (ella actualmente trabaja en Baja Verapaz). La autora ha sido testigo de esta disposición en su experiencia de trabajo con diferentes comunidades y organizaciones del interior del país (Lam, 2011).

En las zonas rurales hay poco o ningún apoyo para vigilancia. Hay casos en que los profesionales se sienten amenazados o desplazados por las autoridades (especialmente cuando comienza un nuevo período gubernamental). Sin embargo, Estrada considera que una estrategia para la innovación representa una oportunidad emocionante para muchas personas y profesionales capaces, con vocación real para sus campos. Esto puede motivarlos a compartir sus conocimientos con profesionalidad, entusiasmo y actitud positiva y brindarles un nuevo espacio para el crecimiento personal, profesional y económico.

\subsubsection{Dirigiendo el Emprendimiento hacia el Sector Salud en Guatemala}

La creación de una estrategia que genere nuevas ideas permitirá a emprendedores, visionarios y organizaciones compartir los resultados, datos y nuevos diseños, por que se requiere la participación de varios sectores. El sector empresarial está relacionado no sólo con los negocios, sino también con políticas legales y gubernamentales, por lo que la autora consideró importante contar con la opinión de un experto en actividades de emprendimiento y otro para el área legal. En esta sección se contó con el análisis de dos profesionales. El primero, con: Licda. Katerin Figueroa, Community Manager, quien trabaja para Mercy Corps, MicroMentor Guatemala. Y el segundo con: Licda. María Carla Herrera Ortega, Abogada y Notaria. 
Como experta en el área empresarial, Figueroa mencionó que hay una necesidad de mejorar la calidad de la educación en el país. Es necesario mejorar el plan de estudios en todas las áreas. En el entorno de Guatemala los centros que promueven la innovación y la investigación o aplican el pensamiento de diseño son casi nulos, se enfocan en teoría, pero no en practicar e innovar. Es necesario reemplazar la "memorización" y enfocarlos al análisis y solución de los problemas.

La creación de una base de datos requiere una gran inversión. Por eso, desde su punto de vista, si no hay un donante extranjero interesado en invertir o aportar, será difícil de lograr. Respalda su opinión con experiencia propia: las estadísticas del país (sobre diferentes temas) son del 2011 (nota: en DCH esto representa un gran desafío de diseño para innovar con la viabilidad de los recursos existentes). Opina que no hay información actualizada sobre temas de salud y otros; y no se debe a la apatía sino a la falta de inversión necesaria para una base de datos actualizada. Y si la estrategia se desarrollara a través del gobierno, sería necesario tener un gobierno basado en las capacidades en lugar de retorno favores.

Figueroa tuvo la oportunidad de compartir sus preocupaciones en cuanto al tema con Melinda Gates, y señaló que en el país, en el tema de la innovación, iniciativas, leyes, políticas y estrategias son inexistentes, todavía es necesario desarrollarlo. Pasar una ley no es fácil y muchas veces todo se hace de acuerdo a los intereses del gobierno. Ella lo experimentó personalmente, al trabajar en política de emprendimiento "engavetada". Política que después de un esfuerzo considerable, fue finalmente firmada y aprobada sólo porque el Gobierno estaba en la necesidad de presentar resultados anuales, no por interés en ella (nota: desde el punto de vista del DCH esta 'necesidad de resultados anuales' también es una oportunidad a explorar).
En el área de experiencia de Figueroa el DCH en este momento gana importancia, pero no precisamente en el área de la salud.

En el análisis jurídico, la Licda. Herrera opina que Guatemala no cuenta con instituciones que tengan como objetivo proporcionar la innovación o el desarrollo de manera correcta. Las ya existentes, están llenas de corrupción, lo que facilita la desviación y robo del dinero, en lugar de proporcionar el servicio necesario para lo que fueron creadas. En su opinión, hay una falta fundamental de la empatía en términos de deuda existente entre las instituciones gubernamentales con las de salud pública y entes privados, lo que se refleja en el mal funcionamiento de todo el sistema.

Desde su punto de vista no hay necesidad de hacer una nueva legislación, sino que es necesario modificar las políticas ya establecidas y adaptarlas a la realidad. Pero lo más importante es la aplicación correcta y eficaz de la ley, sin privilegios, beneficios o favores individuales.

\subsubsection{Despertar del conocimiento de DCH dirigido al Diseño del Modelo de Ingresos}

A partir del hecho de que, es importante para el enfoque de DCH integrar el plan de negocios en el desarrollo del diseño, la autora consideró importante indagar la opinión de expertos en finanzas o los negocios sobre su conocimiento sobre Pensamiento de Diseño e Ingresos Modelo de DCH. Para hacer este análisis se obtuvo la colaboración del Lic. Gerardo Corzo, Director del Departamento de Marketing, UNIS.

A pesar de que el Lic. Corzo no había oído hablar de los términos "Pensamiento de Diseño" o "Diseño Centrado en el Humano" en relación al área financiera, el opina que es un concepto muy interesante.

En su opinión habría que empezar por preguntas como: ¿Qué se requiere para mejorar? 
¿Qué beneficios traería tal estrategia? y otros. Él considera que sería muy importante contar con una amplia información sobre lo que se necesita de cada sector (para iniciar el camino hacia la innovación), y empezar a reunir y compartir los resultados con los diversos sectores que pueden ayudar en este esfuerzo. Esto incluiría medios de comunicación, universidades, individuos, Cámaras de Comercio e Industria, y otros.

Remarcó que sensibilizar a los sectores puede ser difícil. Sobre todo el sector político y gubernamental a causa de la corrupción que prevalece en el sistema, por lo tanto, es necesario crear un plan para atraer su atención y ganar el interés.

\subsection{Puntos Principales de la Estrategia para la Innovación}

Idealmente el desarrollo e implementación de una estrategia de innovación, en términos generales y viables, debería tener como función principal, primero: hacer accesible el conocimiento y la formación sobre la base del pensamiento de DCH. Colocar este conocimiento y su práctica a disposición de los estudiantes universitarios (de todos los campos), los estudiantes de diversificado (especialmente aquellos con especializaciones técnicas), personas independientes que ya están en el campo de la empresa o sector a los empresarios a proporcionar herramientas competitivas. En segundo lugar, la creación de una base de datos, haciendo uso de los recursos disponibles y viables del país.

El ejercicio y el entrenamiento de $\mathrm{DCH}$ requiere el registro de todos los datos de investigación (ya sea durante los cursos de práctica o investigaciones formales) sean colocados de manera organizada, su análisis y posterior retroalimentación, con el fin de apoyar la iteración de ideas. La base de datos debe ser llevada a cabo y estar respaldada por expertos. Esto dará el respaldo para que los datos (publicados y actualizados) sean fiables (con los derechos, obligaciones y conducta ética que la investigación formal implica). En tercer lugar, la creación de un canal (o canales) de retroalimentación para recepción de información y asesoramiento.

La función de este canal sería proporcionar apoyo en el desarrollo de diseños (servicios, productos, soporte), no sólo en términos de orientación técnica, sino también para la actualización constante sobre las estrategias de $\mathrm{DCH}$. Esto es importante, porque allí precisamente es donde radica el movimiento continuo hacia la innovación.

Los resultados de las prácticas de aprendizaje, de proyectos de diseño y su análisis, permitirán el refinamiento de los diseños, con el fin de alcanzar el objetivo de madurez (independientemente de la naturaleza del producto). Los datos reflejados en el GINI World Bank 2014 demuestran que gran parte de la población guatemalteca pertenece al mercado de la Base de la Pirámide del mundo conocido como BoP Market, por sus siglas en inglés, este sector es un punto focal importante para dirigir el esfuerzo innovador.

Una estrategia para impulsar la innovación, debe contener actividades destinadas a abordar el 'Diseño de Sistemas de Servicio del Producto' - PSS por sus siglas en Inglés (Product Service Systems, Jagtap \& Larsson, 2013) relacionados directamente con el mercado de la Base de la Pirámide; entre estas actividades están:

- Adaptar productos y procesos.

- Invertir en la eliminación de las restricciones del mercado.

- Aprovechar las fortalezas de los pobres.

- Combinar recursos y capacidades.

- Participar en el diálogo sobre políticas con los gobiernos, y otras más. 
Figura 17

Ciclo de Innovación Viable en Guatemala

\section{Camino hacia la Innovación}

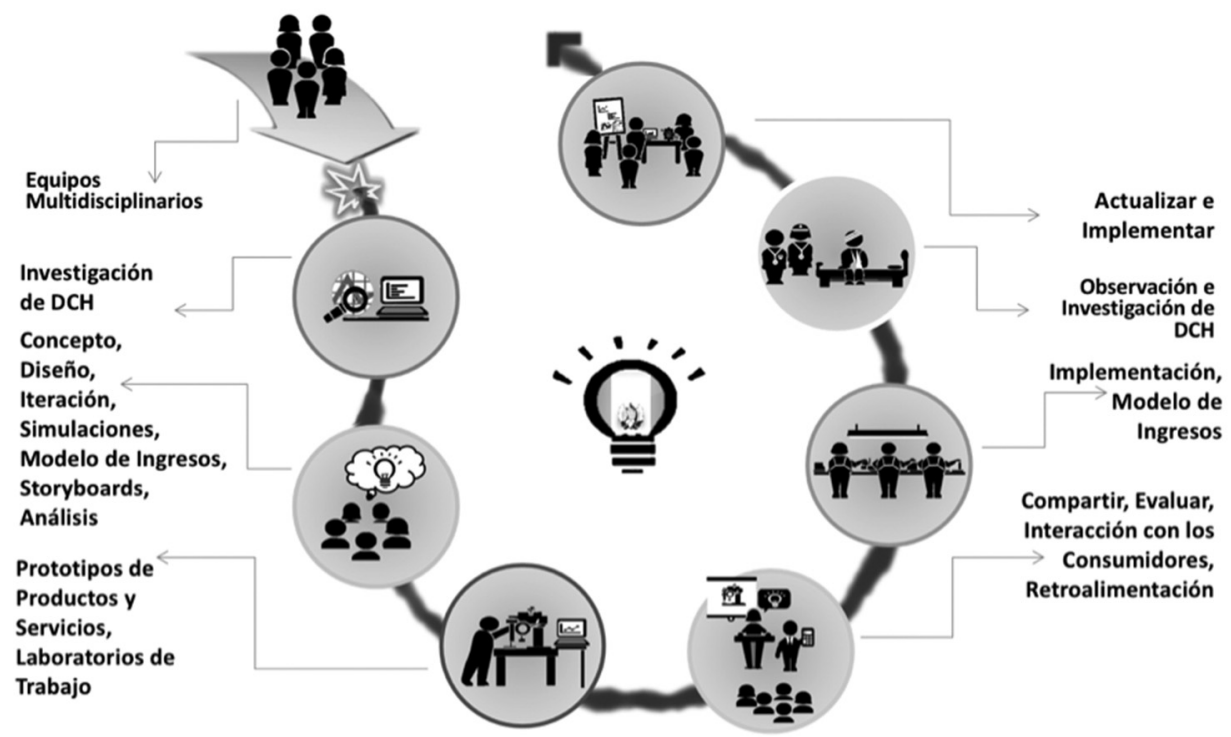

Fuente: Elaboración propia.

Figura 18

Espiral de Innovación hacia la Madurez del Diseño

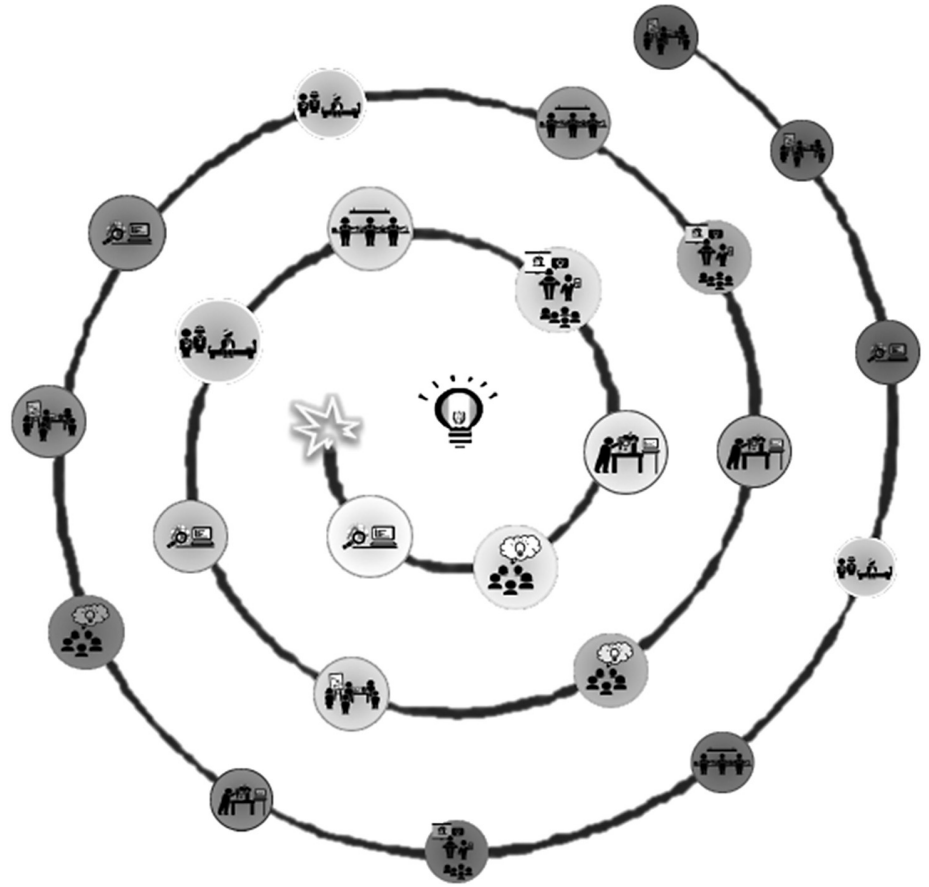

Fuente: Elaboración propia. 


\section{Conclusiones}

El sector de la salud de Guatemala presenta una oportunidad de mercado casi virgen. DCH ofrece soluciones que benefician no sólo el producto a diseñar, sino que abarca todos los aspectos involucrados (medio ambiente, comercial, legal, social y demás). Los temas relacionados con DCH son de poca accesibilidad para los guatemaltecos. El conocimiento de DCH en el ambiente guatemalteco es escaso. Esto es debido a limitaciones tales como costo y tiempo, o simplemente por que se ignora que existe.

Guatemala sufre de altos niveles de corrupción. A pesar de que los acontecimientos políticos y sociales recientes dan esperanza de cambio, el país aún está lejos de lograr un cambio para mejorar la situación de la salud en un futuro cercano. Por lo tanto, es importante tener en cuenta que, mientras un cambio de políticas y ética administrativa de Guatemala se desarrolla, las nuevas generaciones de profesionales están entrando en el ambiente de trabajo sin el conocimiento esencial para embarcarse en proyectos innovadores para el área de la salud.

La autora llegó a la conclusión de que la necesidad de innovar es crucial para el país. Por eso, es muy importante impulsar una estrategia que promueva la práctica del DCH (en nivel diversificado, técnico, universitario y para demás personas interesadas en participar). Básicamente por que $\mathrm{DCH}$ provoca un efecto en cadena hacia la innovación (debido a la naturaleza integral de su enfoque). La necesidad y la oportunidad de diseño es real en todos los aspectos del sector de la salud (ej. un hospital cuenta con todos los servicios y necesidades requeridos en un hotel, pero también incluye todos los aspectos relacionados con la salud: médico, social, terapéutico, y demás).

En países como Guatemala hay necesidad de tener mejores opciones para el cuidado de la salud, no sólo basado en el costo, sino en cali- dad de alto nivel, adecuado a las necesidades de las personas y el medio ambiente 'los consumidores de productos en el mundo en desarrollo no están buscando bajo costo, ni alternativas de baja calidad similares a lo que está disponible para los clientes más ricos. Son un grupo consciente del valor que exige y merece alternativas asequibles que cumplan con un estándar de desempeño competitivo. Una de las cosas más importantes en DCH es la participación de expertos, que provengan de diferentes áreas de experiencia profesional y práctica, para ayudar con la complejidad del trabajo.

Guatemala es un país lleno de historias de personas fuertes y con grandes sueños, que se enfrentan día a día a una dura realidad, una sociedad que es herida por la corrupción constante, violencia y pobreza; sin embargo, una población feliz que se enfoca todos los días a ver el lado positivo de la vida.

Aunque el enfoque de DCH no es infalible, su conocimiento y práctica acumula experiencia, promueve la iteración de las ideas y en general, contribuye al perfeccionamiento y la maduración de los diseños, porque modifica el pensamiento de las personas. Por lo tanto, a través de él se puede lograr una mejora gradual del Sector de la Salud de Guatemala, beneficiar comunidades, y por qué no soñar, contribuir a la mejora de todo el país.

\section{Referencias}

Andrade, J. L. (2014). Conocimientos del personal auxiliar de enfermería que labora en el servicio de sala de operaciones de la emergencia de adultos del hospital Roosevelt acerca de los riesgos laborales a los que están expuestos. Non-Published Work July 2014, from Biblioteca de la Universidad San Carlos de Guatemala.

Andrino, J. M., De León, J. E., López, C. \& Morales, H. M. (2014). Informe Anual Circunstanciado: Situación de los Derechos Humanos y Memoria de Labores 2014 
[Circunstanciado Annual Report: Situation of Human Rights and Labor Report 2014]. Retrieved 2014, from Procuraduría de los Derechos Humanos, Guatemala.

Arias, J. C., Ketelhön, N. \& Martínez, C. (2013). Los Hospitales de Centroamérica [The Hospitals of Central America]. INCAE Business Review. Retrieved January 2013.

Bachman, J. (2014). Fatal Asiana Crash in San Francisco Blamed on Confused Pilots. Bloomberg Business.

BBC Monitoring. (2015). Meet the Facebook reporters of India-Bangladesh enclaves. BBC. Retrieved July 31, 2015, from BBC Asia, http://www.bbc.com/news/ world-asia-33729207

Becerril-Montekio, B. \& López-Davila, L. (2011). Sistema de Salud de Guatemala [Healthcare System of Guatemala]. Retrieved April 24, 2011.

Bendewald, M., Berger, B., Birr, D., Bonnema, E., Chan, C., Coca, S., et al. (2013). Advanced Energy Retrofit Guide for Healthcare Facilities. Retrieved September 2013

Boy, G. A. (2013). Orchestrating Human-Centered Design. London: Springer-Verlag.

Brown, J. M. \& Schmith, N. A. (Eds.) United States of America: Jones and Bartlett Publishers, LLC.

Cain, S. (2013). Quiet, the Power of Introverts in a World that Can't Stop Talking. USA, NY: Crown Publishing Group.

Chow, S. \& Morgan, A. (2007). The Economic Impact of Implementing an Ergonomic Plan. Retrieved May 2007, from www. ncbi.nlm.nih.gov/pubmed/17802997

Courcy's Intelligence. (2015). Guatemala: Human rights ombudsman warns of health crisis.
Cu-Uvin, S. (2015). HIV and HPV: A Double Curse for Women in Resource Constrained Countries. Gender, Health and Environment in Sustainable Development, Institute for Development and Human Security, Ewha Womans University, Sponsored by Bill \& Melinda Gates Foundation. 2nd International Conference, May 11, 2015. Handout p. 2.

De León, J. E. (2013). Informe Anual Circunstanciado: Informe de Situación 2013 [Circunstanciado Annual Report: Progress Report 2013]. Retrieved 2013, fromThe International Ombudsman Institute

Gheorghe, F. \& Van der Loos, M. (2013). Participatory Design for Surgical Innovation in the Developing World. In Chakrabarti, A. \& Prakash, V., (Eds.). ICoRD'13, Lecture Notes in Mechanical Engineering, India: Springer.

\section{Global Entrepreneurship Monitor [GEM].} (2014). A high level of entrepreneurial activity, consisting mainly of poorly capitalizes businesses related to consumption, with low value-added, innovativeness, and growth prospects. Retrieved 2014

Goodman, E., Kuniavsky, M. \& Moed, A. (2012). Observing the User Experience. USA, MA: Morgan Kaufmann Publishers.

Grameen Foundation [GF]. (2015). Human Centered Design, Financial Services. From GF, http://www.grameenfoundation.org/what-we-do/financial-services/ human-centered-design

Grover, A. (2011). Informe del relator especial sobre el derecho de toda persona al disfrute del más alto nivel posible de salud física y mental, Misión Guatemala [Report of the Special Rapporteur on the right of everyone to the enjoyment of the highest attainable standard of physical and mental health, Mission Guatemala]. From United Nations General Assembly 
Guatemala National Team [GEM] (2013). Entrepreneurs in Guatemala. Universidad Francisco Marroquin. Retreived 2013, from http://m.gemconsortium.org/ entrepreneurship/guatemala/

Helliwell, J., Layard, R. \& Sachs, J. (2013). World Happiness Report 2013. http://unsdsn. org/wp-content/uploads/2014/02/WorldHappinessReport2013_online.pdf

Human Factors and Ergonomics Society [HFES]. (2014). Human Factors/Ergonomics Research Prompts Advances in Health Care IT. Human Factors and Ergonomics Society. Retrieved September 16, 2014, from HFES News, https://www.hfes. org/web/DetailNews.aspx?ID=351

Innovation Lab, Las Ideas Transforman. (2015). Leveraged Freedom Chair in Guatemala. (n.d.) From BID Innovation, http://www. bidinnovacion.org/en/proyectos-activos/ proyecto-04/

International Academy for Design and Health. (2015). Design \& Health. Retrieved 2015, from IADH, http://www.designandhealth. com/

International Development Enterprises.org [IDEO]. (2011). HCD Human Centered Design Toolkit. Second Edition. Canada: IDEO.

Jagtap, S. \& Larsson, A. (2013). Design of Product Service Systems at the Base of The Pyramid. In Chakrabarti, A. \& Prakash, V., (Eds.). ICoRD'13, Lecture Notes in Mechanical Engineering, India: Springer

Kelley, D. (2007). Human-centered design. Retrieved May, 2007, form TED

Lam, M. (2011). Organización para la Puesta en Marcha de una Microempresa de Confección, Aplicada a la Asociación Ixil Para el Desarrollo Económico Y Social (AIDES), en Nebaj, Quiche [Organization, Proces- ses and Methods, for the implementation of a micro clothing, addressed to the Ixil Development Association (AIDES) in Acul, Nebaj, Quiché, Guatemala].

Lawnorder. (2003). Accidental Leakage of Cesium-137 in Goiania Brazil in 1987. Retrieved March 3, 2003. Lawnorder, http://lawnorder.blogspot.kr/2003/03/case-study-accidental-leakage-of.html

McCullough, C. Ed. (2009). Evidence-Based Design For Health Care Facilities. United States of America: Sigma Theta Tau International

McKay, C. \& Seltzer, Y. (2014). What Human-Centered Design Means for Financial Inclusion. Retrieved October 16, 2014, from CGAP (the Consultative Group to Assist the Poor)

Ministerio de Salud Pública y Asistencia Social [MSPAS]. (2015). Plan Operativo Annual y proyecto de Presupuesto, Ministerio de Salud Publica y Asistencia Social, Año 2015 [Annual Operating Plan and Budget Project, Ministry of Health and Welfare, 2015]. Retrieved 2015, from portal.mspas.gob.gt

NovoEd. (2015). Take a course! Learn, Collaborate, Innovate. From NovoEd, Inc. https://novoed.com/courses

Ogunlesi, T. (2012). Seven ways mobile phones have changed lives in Africa. CNN. Retrieved September 14, 2012, from CNN Africa, http://edition. cnn.com/2012/09/13/world/africa/ mobile-phones-change-africa/

Phares, E. (2011). Evidence-Based Design in Healthcare Interior Design. Germany, Saarbrucken: Lambert Academic Publishing.

Springer, T. (2007). Ergonomics for Healthcare Environments. Retrieved 2007, from KnoIl.com 
The World Bank. (2014). GINI index (World Bank estimate). Retrieved 2014, from The World Bank, http://data.worldbank.org/indicator/SI.POV.GINI/ countries/1W?display=map

(2015). Guatemala Data. Retrieved 2015, from worldbank.org

Transparency International. (2014). Corruption Perception Index 2014. Retrieved 2014, from Transparency International, https:// www.transparency.org/cpi2014/results

UK Government. (2013). Management and disposal of healthcare waste (HTM 07-01). Retrieved March 2013.
Universidad del Istmo de Guatemala [UNIS]. (2015). FORUM, Formacion Humanistica [Humanistic education, focused on solidarity). From UNIS, http://unis.edu. $\mathrm{gt} / \mathrm{s}=$ Solidaridad

White, T. (2014). What can save the rainforest? Your used cell phone. TED. Retrieved September, 2014

World Health Organization [WHO]. (2013). Neonatal and Child Health Country Profile

(2015). Guatemala: WHO statistical profile. Retrieved January 2015

(2015). World Health Statistics 2015 (p. 153). 